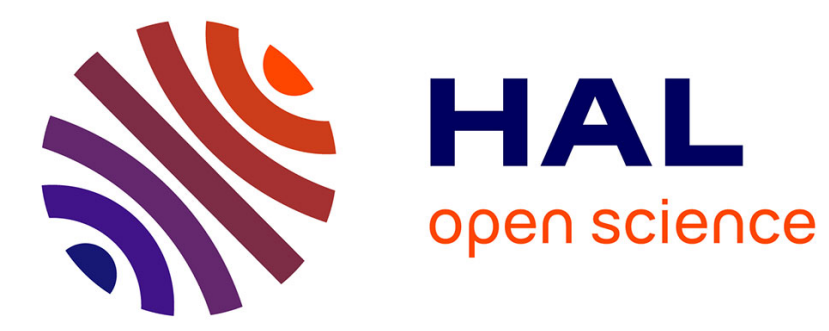

\title{
Technical feasibility of Power Flow Controllers for HVDC grids
}

Joan Sau-Bassols, Florent Morel, Selle Toure, Serge Poullain, Frank Jacquier

\section{To cite this version:}

Joan Sau-Bassols, Florent Morel, Selle Toure, Serge Poullain, Frank Jacquier. Technical feasibility of Power Flow Controllers for HVDC grids. 2021 AEIT HVDC International Conference (AEIT HVDC), May 2021, Genoa, Italy. pp.1-6, 10.1109/AEITHVDC52364.2021.9474591 hal-03282362

\section{HAL Id: hal-03282362 https://hal.science/hal-03282362}

Submitted on 9 Jul 2021

HAL is a multi-disciplinary open access archive for the deposit and dissemination of scientific research documents, whether they are published or not. The documents may come from teaching and research institutions in France or abroad, or from public or private research centers.
L'archive ouverte pluridisciplinaire HAL, est destinée au dépôt et à la diffusion de documents scientifiques de niveau recherche, publiés ou non, émanant des établissements d'enseignement et de recherche français ou étrangers, des laboratoires publics ou privés. 


\section{Technical feasibility of Power Flow Controllers for HVDC grids}

\author{
Joan Sau-Bassols \\ Power Electronics \& Converters \\ SuperGrid Institute \\ Villeurbanne, France \\ joan.saubassols@supergrid-institute.com \\ Serge Poullain \\ Supergrid Architecture \& Systems \\ SuperGrid Institute \\ Villeurbanne, France \\ serge.poullain@supergrid-institute.com
}

florent.morel@supergrid-institute.com

\author{
Sellé Touré \\ Supergrid Architecture \& Systems \\ SuperGrid Institute \\ Villeurbanne, France \\ selle.toure@supergrid-institute.com
}

\author{
Frank Jacquier \\ High Voltage Substation Equipment \\ SuperGrid Institute \\ Villeurbanne, France \\ frank.jacquier@supergrid-institute.com
}

\begin{abstract}
Power Flow Controllers (PFCs) or Current Flow Controllers (CFCs) are expected to be required in future High Voltage Direct Current (HVDC) grids to aid in the current distribution. This work analyses the feasibility of this kind of devices from the implementation point of view. First, the needs of a PFC as a medium voltage converter floating at high voltage are assessed. Then, the paper discusses the challenges to implement one of these converters, such as the sizing of the power electronic elements, cooling management, insulation from ground, etc. Most of them are already tackled in other existing devices in the field of high voltage. Powering the PFC semiconductors gate-drivers and other auxiliary systems at high potential is seen as a particular point to take into account when building the device.
\end{abstract}

Index Terms-power flow controller, current flow controller, HVDC grid, feasibility, physical implementation

\section{INTRODUCTION}

High Voltage Direct Current (HVDC) grids are seen as a key factor to facilitate the integration of renewable power sources and they can also increase the flexibility of the power system [1]. However, they pose several challenges in terms of control, operation, protection, etc. [2]. Power flow control inside the HVDC grid becomes a concern since the converter stations do not provide enough degrees of freedom to avoid congestions in the lines [1].

That is the reason why additional converter devices known as Power Flow Controllers (PFCs) or Current Flow Controllers (CFCs) are expected to be installed in certain nodes of the DC grids to aid in the power flow regulation [3]. These devices can be understood as the equivalent of Flexible AC Transmission Systems (FACTS) but applied to HVDC grids [4]. Industry and academia have suggested numerous circuits to act as PFCs, providing electrical diagrams to illustrate the concepts. The proposed options can be classified as: series switching resistors [5], full power converters connected between poles and variable voltage sources inserted in series with one line [6] or with several lines [7], [8]. Most of the works are supported by simulations of the power electronic circuits [9] and some down-scaled converters have been built in laboratories to test the concepts [10], [11]. Several control schemes for these converters have been also proposed and their stability inside the HVDC grid has been analyzed [9], [12]. Nevertheless, there are few examples of works focusing on the physical implementation of those devices (for example the sizing of the power electronic elements [13]) and the associated auxiliary equipment that will be required. The feasibility of those converters is then questionable.

This paper analyses the technical feasibility of PFCs, without going into detail in a particular converter topology. The first section explains the reasons of focusing the work in PFCs connected in series with several HVDC grid lines (DC/DC converters). The needs and requirements of these devices, from the implementation point of view, are identified. Then, the paper discusses each of the challenges to build such a device. This work analyses the different aspects to implement the converter (for instance, power electronic switches, cooling management, protections, etc.), while relating them to existing technologies in order to assess which of them are feasible and which of them are possible locks.

\section{NEEDS AND REQUIREMENTS OF A POWER FLOW CONTROLLER}

This section analyzes what are the needs and requirements of power flow controllers in terms of the implementation point of view.

There are several PFC solutions that insert a variable voltage in series with the lines in order to control the line currents. A first option consists in exchanging power with the $\mathrm{AC}$ grid with an AC/DC converter [6]. However, it requires an AC transformer to interconnect the AC and DC grids, which can increase the cost of the solution. DC/DC converters connected between the two poles on one side and then in series with one pole on the other side are another possibility [14]. Their main drawback is the need of a converter able to withstand the 
pole-to-pole voltage, which implies a high number of power electronic switches.

This work focuses on a third option, the use of seriesconnected PFCs that exchange power between two [7] or more DC lines [10]. They are implemented as DC/DC converters (also called interline PFCs) and appear to be a more costeffective solution than the previous alternatives due to the lack of the AC transformer or the high voltage converter.

The aforementioned PFCs exchange power between the lines where they are connected, what allows them to insert series voltages to control the currents through the conductors [9]. They are connected in series with the lines, floating at the positive or negative pole. Thus, they do not withstand the poleto-ground voltage, but the totality of the line current circulates through them.

The voltage that they need to insert to control the currents depends on the scheme of the HVDC grid where they are installed and the resistance relation between the conductors in this grid [3]. In the literature it is possible to find PFCs used to control the currents of HVDC grids with pole-toground voltages in the order of hundreds of $\mathrm{kV}$, while the PFCs themselves insert voltages of a few $\mathrm{kV}$. On the contrary, the currents that circulate through the components of the PFC are up to $2 \mathrm{kA}$ (for cables) or up to $4 \mathrm{kA}$ (overhead lines) [9].

In order to provide a specific example, the work in [13] considers the installation of a PFC connected between two lines of Zhangbei grid in China, a 4-terminal $\pm 500 \mathrm{kV}$ bipole [15]. An hypothetical upgrade of the converter station S3 is assumed, which causes the overload of one of the grid lines (red line in Fig. 1). In order to redirect the current flow to an underused line, the PFC has to insert voltages in series up to $6 \mathrm{kV}$. The maximum current that flows through the device is in the order of $3 \mathrm{kA} \mathrm{[13].}$

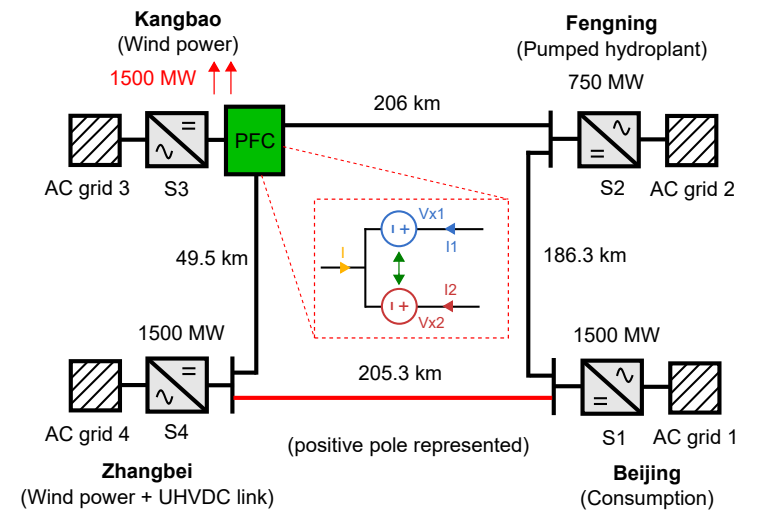

Fig. 1. Example: scheme of the Zhangbei grid with a PFC installed in node 3 in order to prevent the overload of line 41 (in red) after the power upgrade of station 3 .

The PFC is expected to be a converter for medium voltage (some $\mathrm{kV}$ ) with a considerable current rating (some $\mathrm{kA}$ ), leading to a power rating of some tens of MW. However, the insulation of the device must be in the order of the pole-toground voltage (some hundreds of $\mathrm{kV}$ ), since it is floating at the positive or negative pole. Fig. 2 illustrates, two PFCs connected between a converter station and two generic lines 1 and 2. It is important to notice that in order to keep the symmetry of currents between the positive and negative pole, a PFC on each pole is required.

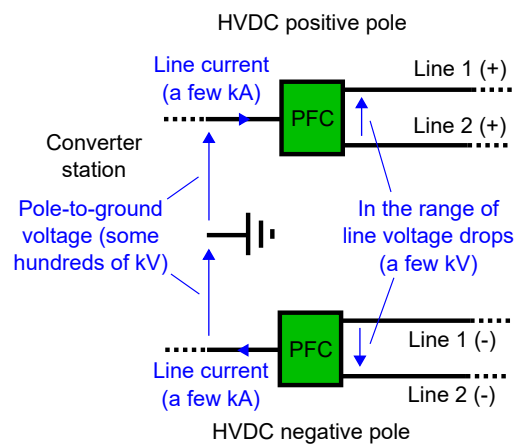

Fig. 2. Generic diagram of two PFCs installed in a bipole or symmetrical monopole between a converter station and two lines.

In terms of power electronic elements, several diodes and controllable switches with or without current and voltage reversibility are needed. Their number and configuration will depend on the selected converter topology, which in turn it will depend on the required voltage and current bidirectionality of the PFC inside the grid. Two examples of PFC converters with different capabilities in terms of bidirectionality, passive components and switches are presented in Fig. 3.

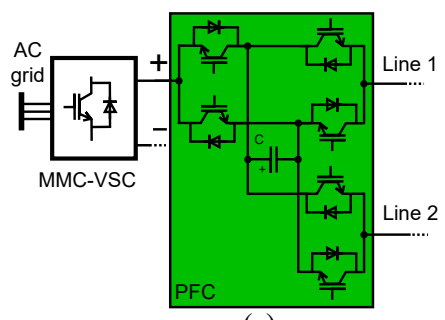

(a)

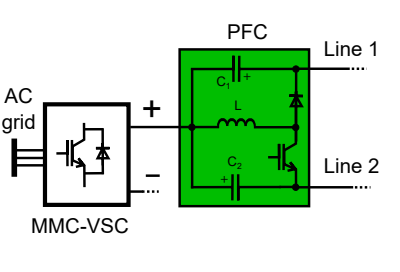

(b)
Fig. 3. PFC converter structures (the controllable switches are represented as Insulated Gate Bipolar Transistors (IGBTs) for the sake of simplicity). (a) PFC proposed in [7] with one capacitor. (b) PFC proposed in [8] with two capacitors and one inductor.

Due to the large currents circulating through the device, it is expected that a cooling system for the power electronic switches may be required, which needs to be compatible with the high voltage insulation.

In addition, the converter also requires the corresponding control hardware (gate-driver boards, controller boards, etc.) and voltage and current sensors to properly operate the device. The control systems must be able to communicate with the operator of the HVDC grid in order to provide the adequate orders to the PFC (bypass, insertion, current setpoints, etc.). In addition, the PFC converters at each pole may need to be synchronised.

The elements that need to be as close as possible to the PFC converter, such as gate-drivers, fast control, possibly cooling an the other auxiliary systems, must be powered while being at the high voltage potential. The elements that do not need to be as close to the PFC converter can be located at ground level and properly insulated from the PFC converter.

The DC grid may not require permanently the operation of the PFC, which means that the PFC converter should incorporate bypass switches to be able to insert or bypass 
it according to the requirements of the Transmision System Operator (TSO) or the operator of the HVDC grid. This bypass or insertion should be performed without interrupting the power transmission. In a similar manner, it must be ensured that during PFC maintenance works, the PFC converter must be properly grounded and the rest of the grid must be able to operate without interruption.

Being a power electronic device that has to be installed in an HVDC grid, the PFC increases the number of elements that can be susceptible to fail in the overall system. Because of it, the converter has to be properly integrated into the grid ensuring that a failure in one of its components or a fault in the converter do not compromise the grid power transmission. Specifically, a PFC exchanging power between two lines or more has to be installed in the common point of connection of three lines at least (one of the lines can be also an MMC station). This implies, that a failure or a fault in the PFC can bring severe consequences to the HVDC grid, highlighting the importance of its proper integration.

On the contrary, if a fault happens in the HVDC grid, the PFC must be also protected against possible voltage and current surges. Some works consider the integration of PFCs with DC Circuit Breakers (DCCBs) [16], meaning that the two functionalities are merged in a single device with the save in switches and equipment that this can bring. Although it is an attractive idea, for the sake of simplicity, this work considers the first hypothesis, that the PFC needs to be protected from faults in the HVDC grid.

Finally, the PFC, as an element that modifies the current inside the HVDC grid, has to be coordinated in terms of operation and control with the grid where it is installed. The stability and the absence of undesired interactions have to be ensured.

\section{Challenges OF BUILding A POWER FlOW CONTROLLER}

This section presents the challenges to build the PFC in order to comply with the needs and requirements introduced in the previous section. It is assumed that the PFC will be added into the existing HVDC system, where the converter stations (probably MMC) have already been built. Thus, this work considers that it is not installed in the valve hall of the existing converter stations but in a separate platform.

\section{A. Sizing of power electronic switches and passive compo- nents}

The PFC is expected to be a medium voltage converter that should be able to apply up to some $\mathrm{kV}$ and conduct a current up to some kA. Depending on the selected PFC topology, those values are translated differently in requirements for the power electronic switches. Taking for example the converter structure in [8] (see Fig. 3(b)) and the case study in [13], the required DC blocking voltage and DC current required for the switches are $6 \mathrm{kV}$ and $3 \mathrm{kA}$, respectively.

For the controllable switches, it is expected to use IGBTs or other alternative devices as Integrated Gate-Commutated
Thyristors (IGCTs). Regarding nowadays technology, the constraints are expected to come from the side of controllable devices (IGBTs or IGCTs) rather than from diodes.

Focusing on IGBTs, on one hand, the maximum blocking voltage of the available IGBTs in the market is around $6.5 \mathrm{kV}$ (a value that diminishes to less than $4 \mathrm{kV}$ to ensure a nominal DC voltage for a 100 FIT failure rate) [17]. This implies that some devices in series may be expected, making opportune the use of press-pack devices. Other more complex converter structures (for example with submodules) could be envisaged in order to get rid of this requirement.

On the other hand, regarding the current of some kA (3 kA for the example in [13]), this is in the same order of magnitude than the maximum DC current rating of the IGBTs found in the market (3 kA) [17]. This possibly implies that the paralleling of devices is required to withstand the current. Another alternative is the use of interleaved structures as in [13], which not only allows to work with the needed current, but it offers advantages in terms of the sizing of the passive components (since the equivalent frequency that they see is higher). Fig. 4(a) presents a possible converter structure of the topology introduced in [8]. The result of considering an interleaved structure for the converter in Fig. 4(a) is shown in Fig. 4(b) with two interleaved branches (doubling the number of IGBTs, diodes and inductors with the half of the current rating compared to the initial circuit).

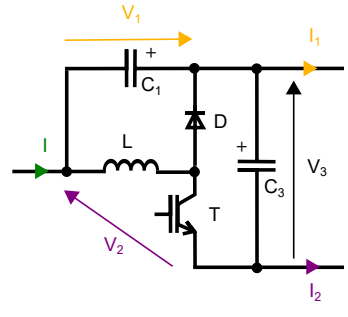

(a)

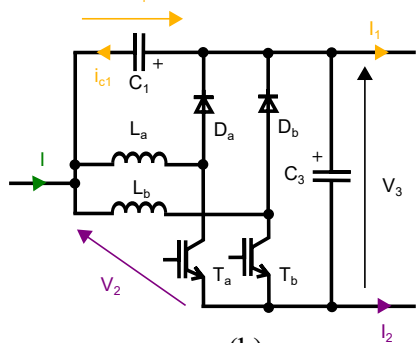

(b)
Fig. 4. (a) PFC structure introduced in [8]. (b) PFC structure introduced in [8] with two interleaved branches.

Using IGCTs provides a similar voltage rating but a higher current capability during faults. They also offer a reduced onstate voltage drop (lower conduction losses) when compared to IGBTs. However, they come with some disadvantages, for instance, the need of an additional snubber circuit to limit the switching speed and protect the associated freewheeling diode when the IGCT turns on [17]. Also, their maximum switching frequency is expected to be lower when compared to IGBTs. By increasing the switching frequency of the PFC, the switching losses may increase and the use of IGCTs may not be an option, but the size of passive components diminishes.

Regarding the passive components, they will depend on the selected topology. Most of the converters include one or more capacitors [7], [10] in order to be able to insert voltages in series with the lines. Some structures include also inductors [8] and/or AC transformers. For instance, the converter in [13] requires two capacitors and one inductor (see Fig. 4(a)). For the capacitors, the high current need can lead to use several commercial capacitors connected in parallel. Regarding 
the inductor, the current requirement can lead to inductors with considerable size and weight compared to the other PFC elements, but still being feasible and made on-demand.

Considering the requirements in [13] and the converter structure in Fig. 4(b)), Fig. 5 presents a 3D design of the main power electronic devices and passive components of the PFC converter.

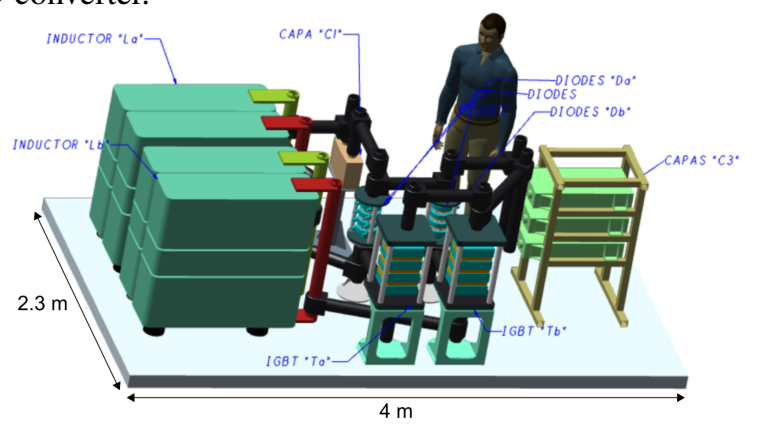

Fig. 5. 3D design of the power electronic devices and passive components of a PFC [13] (the capacitors on the shelves). The image illustrates the size of the elements but it does not provide a definitive arrangement. It does not take into account the insulation distances, optimum location or the definitive connection between elements (black bars). More elements as cooling, control systems, etc. are expected to be present, which are not depicted.

\section{B. Cooling management}

According to [13], the total losses of the PFC are expected to be around $100 \mathrm{~kW}$. Some other works in the literature report values in the same order of magnitude [7], [10]. Although, it corresponds to a lower order of magnitude than the losses in the lines (around $5 \mathrm{MW}$ in [13]), it is a non-negligible value for a medium voltage converter. The calculated losses are up to $8 \mathrm{~kW}$ for a single semiconductor $\left(\approx 10 \mathrm{~W} / \mathrm{cm}^{2}\right)$ for the case study in [13]. This represents a high value of power losses per switch, which may require water-cooling systems with the corresponding cold plates, pipes and the water cooling unit. The PFC adds the challenge that at least the cold plates in contact with the semiconductors will be at the voltage of the pole. The rest of the cooling system can be located at the same platform at the high potential or it can be located at ground level using deionized water to cool the semiconductors. The first option may simplify the design of the cooling system but it can increase the size and cost of the platform (due to the pumps) and it can also increase the power to be fed to the devices at pole potential (refer to Section E). The second option allows to reduce the size of the platform but requires the proper insulation between the different elements of the cooling system. It is not seen as a lock since it is structurally similar to the cooling system used in HVDC systems (for example in MMC submodules), where the cooling unit is at ground level and the submodules are cooled using pipes of deionized water [18]. An alternative option to cool the devices can be the use of a thermosiphon, a passive solution (pump-less) without pole-to-ground insulation requirement [19].

\section{Insulation from ground}

As mentioned in Section II, the PFC will require a high voltage insulation from ground (some hundreds of $\mathrm{kV}$ ). It is expected that the converter will be placed on a platform supported by insulators for this voltage level. This concept is similar to the one used for series compensators in AC systems, as Fixed Series Compensation (SC) or Thyristor Controlled Series Compensation (TCSC) [20]. The question of insulation can be dealt with similar technologies. DC insulation can be different from AC, but solutions exist for HVDC converters as valves at high voltage and DC reactors at pole potential have to be also supported and insulated from ground.

\section{Installation in a tank}

Some elements of the PFC cannot be exposed to the environment. That is the case of the power electronic elements and the corresponding control boards that should be enclosed in a cabinet.

In series-connected FACTS platforms, the semiconductors and the control boards are placed in a cabinet while the surge arresters, capacitors and inductors (air core) are placed outside without protecting them from the environment [20].

For the PFC, the capacitors may need to be very close to the semiconductors in order to diminish the stray inductances. In case of inductors, depending on the physical implementation (air core, iron core, etc.) their requirements might be different. Air core inductors can be located outside (such inductors exist for some MMC stations), but iron core inductors are expected to be protected from the environment. Therefore, a possible alternative is to enclose all the PFC in a tank to protect the elements from the environment. This can be more costly if the whole platform needs to be covered but it is not seen as a lock.

\section{E. Powering the devices}

In order to operate, several PFC elements require to be powered. This is the case of the gate-driver boards, control systems, cooling management (in case of being on the platform), etc.

Powering those elements can be a challenge since the different devices will be on a platform floating at high voltage, with important insulation from ground. Two approaches are identified:

1) Harvesting energy from the environment: In this case, energy is extracted from the surroundings of the elements that need to be powered (at pole potential). One possibility is the approach used in the MMC submodules, in which the voltage of the submodule capacitor is used to power the gate-driver boards for the semiconductors [18]. A similar strategy can be used for the PFC topologies that have a capacitor with a constant voltage [7] and that is charged if the switches are not powered (start-up). If the power consumption is high (for instance, the cooling management is on the platform), this option may not be reasonable. Other converter structures with capacitors modifying their voltages depending on the operating point may pose more challenges to implement this approach [8] (when their voltages are close to 0). Other options consisting in installing an auxiliary battery with solar panels or small wind turbines could also be considered. [21] proposes a turbine that uses the fluid of the cooling system to provide power to auxiliary elements. 
2) Transfer energy from a source at ground potential: Another strategy can be to transfer the power from a power source at ground potential to the platform at high potential (with the proper insulation). The first option would be an AC transformer, but the high voltage insulation would make it bulky and probably not cost-effective. Some research is being done with optic concepts which are able to provide some tens of W [22] to insulated systems. Pneumatic options as aircompressed generators of $12 \mathrm{~W}$ can be found in the market [23] (they would require an additional air-compressed circuit at ground level). Also, another option could be the use of a coupled electric motor and electric generator by means of an insulated shaft (one device on the platform and the other at ground level). Finally, some proposals of magnetic power transfer can be found in [24], [25] which are based on dominoresonators, and they could also be considered to transmit low power levels.

\section{F. Bypass switches and integration into a bus bar}

As highlighted in Section II, the integration of the PFC is key to ensure the power transmission when the device suffers a failure or it is not needed.

Therefore, it is expected that the device will require bypass switches that allow to insert and bypass the device. Those switches are expected to be mechanical switches in order to have low losses when they are in conduction and the PFC is not necessary. Additionally, they will need to switch the nominal current into the PFC and vice versa, meaning that their commutation requirements will be lower than a DCCB but much higher that disconnectors. The HVDC transfer switches can be candidates to implement those bypass switches [26].

Depending on the type of HVDC grid where it is connected, the PFC can be integrated into a single or double bus bar. The second option allows higher redundancy in case of a fault in one of the bus bars. However, it also increases the investment cost since more switches are needed (see Fig. 6). The type of switches to be used in the bus bar (slow/fast breakers, disconnectors, etc.) will depend greatly on the protection strategy of the HVDC grid.

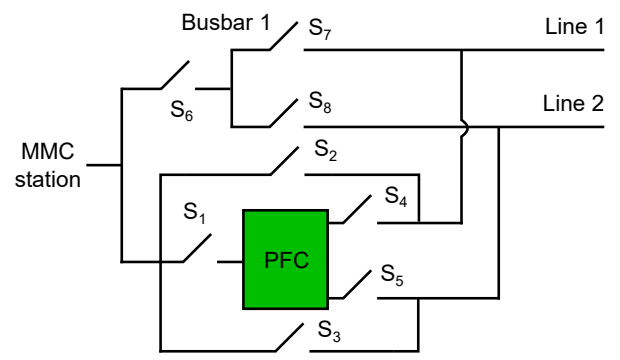

Fig. 6. Scheme of a PFC integrated into a double bus bar, being $S_{2}$ and $S_{3}$ the bypass switches.

\section{G. Protection}

As mentioned before, protecting the PFC against surge voltages and currents due to different kinds of faults is necessary. The mechanical bypass switches introduced in the previous subsection may not be fast enough to protect the PFC from the large currents after the fault, so that additional concepts are needed to ensure the survival of the converter. Several works in the literature propose the installation of additional devices or control schemes to protect the converter from those abnormal situations.

Reference [11] suggests two bidirectional bypass switches (based on power electronics) at each side of a dual H-bridge PFC [7] that are used to divert the fault current from the PFC IGBTs during a DC fault.

The work in [27] proposes a process and additional devices to protect a generic PFC. The process consists in blocking the controllable switches as soon as the fault is detected and in triggering the bypass devices with high surge current capability (thyristors) in parallel with the uncontrollable power electronic switches (normally diodes) in order to divert the high transient current from devices which are not able to withstand it. It also considers to add surge arresters in parallel with the PFC voltage sources (capacitors) and/or across the PFC itself. Fig. 7 shows a possible protection scheme based on this idea.

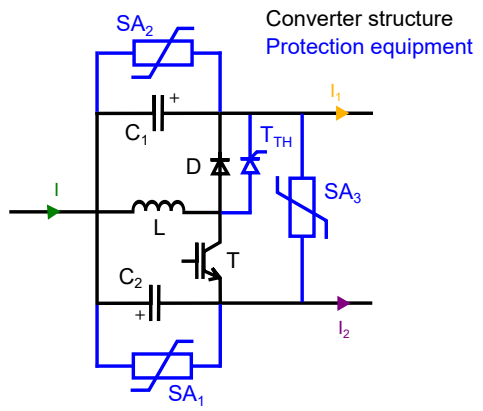

Fig. 7. Scheme of a PFC with the protection equipment in [27]

\section{H. Sensors and communications}

In order to operate the PFC, several sensors to measure voltages and currents will be required. They do not pose a big challenge since there are similar sensors to those ones in series AC compensators and in existing HVDC systems.

In order to measure the currents circulating through the PFC, a similar approach to series AC compensators could be considered where optical current sensors are installed on the platform [20].

Optical current sensors can measure both AC and DC currents and use optical fiber as the sensing element. Hence, they provide inherent insulation which is important for high voltage applications. They commonly rely on the Faraday effect and several manufacturers, such as $\mathrm{ABB}$ or GE, provide this kind of technology [28]. These sensors can be installed in the PFC platform and they can send the optic signals using the fiber optics to the controller station at ground potential.

Regarding the HVDC pole-to-ground voltage measurement, if it is needed, the state of the art is based on RC dividers [28]. In case of requiring the measurement of the PFC capacitors (some $\mathrm{kV}$ at high potential), a similar approach to the one applied to the capacitors of the MMC submodules can be considered (also some $\mathrm{kV}$ at high potential), where their voltage is measured and sent to the controller at ground level via fiber optics [18]. 
The communications with the PFC elements in the platform can be done using fiber optics links similarly to the system used for the MMC submodules.

\section{Control and stability of the converter and the grid}

Finally, the control and stability of the converter and the grid must be guaranteed. The PFC is an element that introduces new dynamics in the HVDC grid and it can potentially modify the DC grid stability. There are several works in the literature that consider a small signal modelling of the HVDC grid and the PFC in order to design a control to fulfill a certain time response while keeping the system stability [9]. Additionally, [12] assesses the stability of the PFC inside the HVDC grid taking into account the passive components of the device (for this case, the capacitance of the PFC in [7]). This highlights that it is important to take this into consideration during the design stage of the PFC converter, but it is probably not a lock to implement the device.

\section{CONCLUSIONS}

This paper has analyzed the technical feasibility of building a PFC. The PFC is expected to be a medium voltage converter, rated for the nominal $\mathrm{DC}$ current of the lines requiring a high voltage insulation (located in an insulated platform or tank). It shares many features with series AC compensators and MMC submodules. The work has showed that the device is feasible in terms of the power electronic elements and they should be taken into account when designing the control and ensuring the system stability. The losses in those elements require cooling systems adapted to the high voltage insulation which can be similar to MMC submodules. Sensors and communications do not seem to pose a challenge either, based on the existing HVDC installations. More work should be focused on how to integrate the PFC into the HVDC grid, in terms of bypass switches, in order to ensure an optimum integration according to the protection strategy. Finally, the powering of the gate-drivers and other auxiliary equipment may pose more difficulties if the approach employed in MMC submodules cannot be used and the required power is high.

\section{ACKNOWLEDGMENT}

This work was supported by a grant overseen by the French National Research Agency (ANR) as part of the "Investissements d'Avenir" Program ANE-ITE-002-01.

\section{REFERENCES}

[1] D. Van Hertem, O. Gomis-Bellmunt and J. Liang, HVDC Grids: For Offshore and Supergrid of the Future. IEEE Press Series on Power Engineering, Ed. John Wiley \& Sons, 2016.

[2] D. Van Hertem and M. Ghandhari, "Multi-terminal VSC HVDC for the European supergrid: Obstacles", Renewable and Sustainable Energy Reviews, vol. 14, issue 9, pp. 3156-3163, Dec. 2010.

[3] E. Veilleux and B. Ooi, "Power flow analysis in multi-terminal HVDC grid," in Power Systems Conf. and Expo. (PSCE), 2011, pp. 1-7.

[4] O. Gomis-Bellmunt, J. Sau-Bassols, E. Prieto-Araujo and M. CheahMane, "Flexible Converters for Meshed HVDC Grids: From Flexible AC Transmission Systems (FACTS) to Flexible DC Grids", IEEE Trans. on Power Del., vol. 35, no. 1, pp. 2-15, Feb. 2020.

[5] Q. Mu, J. Liang, Y. Li and X. Zhou, "Power flow control devices in DC grids," in Power and Energy Society Gen. Meeting IEEE, 2012, pp. 1-7.
[6] E. Veilleux and B. Ooi. "Multi-terminal HVDC with thyristor powerflow controller", IEEE Trans. Power Del. , vol. 27, no. 3, pp. 1205-1212, Jul. 2012.

[7] C. Barker and R. Whitehouse, "A current flow controller for use in HVDC grids," in IET Int. Conf. on AC and DC Power Trans. (ACDC), 2012, pp. 1-5.

[8] S. Touré, F. Morel and S. Poullain, "Power flow control device for controlling the distribution of currents in a mesh network", WO 016449 , 2019.

[9] J. Sau-Bassols, E. Prieto-Araujo and O. Gomis-Bellmunt, "Modelling and control of an interline Current Flow Controller for meshed HVDC grids," IEEE Trans. Power Del. vol. 32, issue 1, pp. 11-22, Feb. 2017.

[10] J. Sau-Bassols, R. Ferrer-San-José, E. Prieto-Araujo, O. GomisBellmunt, "Multiport interline current flow controller for meshed HVDC grids", IEEE Trans. on Ind. Electr. vol. 67, issue 7, pp. 5467-5478, 2019.

[11] S. Balasubramaniam, C. Ugalde-Loo, J. Liang, T. Joseph, R. King and A. Adamczyk, "Experimental Validation of Dual H-Bridge Current Flow Controllers for Meshed HVdc Grids," IEEE Trans. Power Del. vol. 33, issue 1, pp. 381-392, Sep. 2017.

[12] P. Wang, S. Feng, P. Liu, N. Jiang and X. -P. Zhang, "Nyquist stability analysis and capacitance selection for DC current flow controllers in meshed multi-terminal HVDC grids," CSEE Journal of Power and Energy Systems, vol. 7, no. 1, pp. 114-127, Jan. 2021.

[13] J. Sau-Bassols, F. Morel, S. Touré, S. Poullain and F. Jacquier, "Methodology to obtain the specifications and perform the sizing of a power flow controller for meshed HVDC grids", unpublished (provisionally accepted in EPE 2021).

[14] K. Rouzbehi, S. S. Heidary Yazdi and N. Shariati Moghadam, "Power Flow Control in Multi-Terminal HVDC Grids Using a Serial-Parallel DC Power Flow Controller", in IEEE Access, vol. 6, pp. 56934-56944.

[15] G. Tang, H. Pang, Z. He, X. Wei, "Research on Key Technology and Equipment for Zhangbei 500kV DC Grid", The 2018 International Power Electronics Conference (IPEC ECCE Asia), pp. 1-9, Niigata 2018.

[16] A. Mokhberdoran, O. Gomis-Bellmunt, N. Silva and A. Carvalho, "Current Flow Controlling Hybrid DC Circuit Breaker," IEEE Trans. Power Electro. vol. 33, issue 2, pp. 1323-1334, Feb. 2018.

[17] A. Boutry et al., "Figures-of-Merit and current metric for the comparison of IGCTs and IGBTs in Modular Multilevel Converters," 2020 22nd European Conference on Power Electronics and Applications (EPE'20 ECCE Europe), Lyon, France, 2020, pp. 1-10.

[18] K. Sharifabadi, L. Harnefors, H. P. Nee, S. Norrga and R. Teodorescu, Design, Control, and Application of Modular Multilevel Converters for HVDC Transmission Systems. Wiley-IEEE Press, 2016.

[19] M. E. Moustaid, V. Platel and C. Buttay, "Study of convective condensation in a thermosiphon loop,"in 14th International Conference on Heat Transfer, Fluid Mechanics and Thermodynamics (HEFAT 2019) 2019, Wicklow, Ireland, pp. 1-6.

[20] ABB, "Series Compensation Boosting transmission capacity," Technical brochure, [online] 2021.

[21] O. Apeldoorn, M. Cimino, D. Cottet and G. Schuetz, "Supplying auxiliary electrical power in a power electronic device", WO 2013 056933 A3, 2013.

[22] S. Heinig, K. Jacobs, K. Ilves, S. Norrga and H. P. Nee, "Auxiliary Power Supplies for High-Power Converter Submodules: State-of-the-Art and Future Prospects" 2020 [online].

[23] L. Ghossein, F. Morel, H. Morel, P. Dworakowski, "State of the Art of Gate-Drive Power Supplies for Medium and High Voltage Applications, PCIM Europe 2016, May 2016, Nuremberg, Germany.

[24] O. Fabregue and F. Morel, "High-votlage electric insulator", WO 2017 109320 A1, 2017.

[25] J. Qu, L. He, N. Tang and C. -K. Lee, "Wireless Power Transfer Using Domino-Resonator for 110-kV Power Grid Online Monitoring Equipment," IEEE Transactions on Power Electronics, vol. 35, no. 11, pp. 11380-11390, Nov. 2020.

[26] Cigré TB 683 Technical requirements and specifications of state-of-theart HVDC switching equipment, JWG A3/B4.34, 2017.

[27] S. Poullain, F. Morel and S. Touré, "System comprising a power flow control device used to control the distribution of currents in a grid network and means for protecting said device" WO 002266, 2020.

[28] D. Tzelepis et al., "Voltage and Current Measuring Technologies for High Voltage Direct Current Supergrids: A Technology Review Identifying the Options for Protection, Fault Location and Automation Applications," in IEEE Access, vol. 8, pp. 203398-203428, 2020. 